\title{
An Approach to Building Object Models with UML in Embedded Systems
}

\author{
Mohamed T. Kimour and Djamel Meslati \\ Laboratory of Research on Computer Science (LRI), University of Annaba, Annaba, Algeria
}

\begin{abstract}
The UML-based development methods of embedded systems are use case-driven. In these methods, identifying objects that constitute the software system is a critical and hard task, since there is no firm guidelines. In this article, we propose a systematic approach to building object models in embedded systems. After hierarchically decomposing the system into its parts, the approach consists of firstly converting the use case into a statechart that models states of the concerned system parts and, secondly, identifying the objects from the statechart. The proposed approach bridges the gap between the outside behavioral system description, as offered by use cases and the system structure represented by the object model.
\end{abstract}

Keywords: embedded systems, object models, statecharts, UML, use cases.

\section{Introduction}

A strongly increasing application area for realtime systems are embedded systems, which received their name because they are embedded in a technical process. In the development of these systems, there is a growing recognition of the requirements engineering as the initial and possibly the most important activity, where the real demands to be placed on the system have to be identified and captured in a consistent and unambiguous manner $[1,2]$.

The most notable UML-based approaches for real-time system development are based on use cases to capture requirements $[3,4,5,6]$. Use cases are the first artifacts to be established in the software development process. They describe interactions between the system and its environment, and thus capture the functional requirements of the system. Functional requirements are defined in terms of actors and use cases. In the next phases, namely analysis and design, static and dynamic models of the system are developed. The static model defines the structural relationships among domain classes, which are covered by the UML object and class diagrams [7]. The dynamic model describes the system behavioral aspects. In particular, the use case model specifies the functionality of the system, whilst the object diagram specifies the structure of the system. The latter is used as the foundation for the design and implementation phases $[8,9]$.

Once the use case diagram has been built, the developer must identify the objects and classes that describe the system under development. The UML object diagram, sometimes referred to as instance diagram, is useful for exploring real world examples of objects and the relationships between them. This diagram provides a conceptual description of the entities in the application domain. It complements use cases in describing requirements and provides an initial architecture that first captures these requirements. It is an important model being used at almost all steps of the system development activities. For example, behavioral diagrams, such as interaction model, cannot be constructed without knowing the concerned objects [10]. This is why it is fundamental to precisely identify these objects early in the system development process.

Nowadays, there are many methods dealing with the problem of objects and classes identification $[3,8,9,11,12]$. Unfortunately, these methods suffer from at least two drawbacks: first is a focus on classes rather than objects and, second, a direct identification of classes and/or objects from the use case textual description. 
However, when developing an embedded system, it is easier and more important to start with building the object model instead of the class model [8]. Glinz et al. [13] state that class models are inappropriate when more than one object of the same class is used in a specific situation. This is the case with the embedded systems where the elements that do constitute these systems are concrete entities that can be directly mapped to objects. In contrast, classes are the templates that are used for behavioral sharing purpose and do not correspond to concrete elements. Moreover, classes' emphasis is put on commonalities, whereas objects in the embedded systems are often specific.

On the other hand, transition from use cases to objects is not straightforward, because there is no direct one-to-one mapping from use cases to objects. Moreover, identification of objects depends on the use cases representation. Impreciseness, ambiguities, and inconsistency may be present in the textual description of use cases. These description drawbacks make the object identification process difficult and usually resulting in unsatisfactory object or class model.

Current methods that are dedicated to object and/or class identification can be classified into two categories. In the first one, the methods are based on linguistic analysis of the requirements document, which is written in a natural language. Objects and attributes correspond to the nouns, and the operations are related to verbs. In the second category, some tools that help automate the transition from use cases to class and object diagrams are proposed. However, they only can treat a very restricted form of use cases. For both, the main problem is the vagueness, ambiguity and inconsistency of the natural language.

Therefore, transforming the use cases, which divide the system in a functional way, into objects and their properties (attributes, operations), needs some practical guidelines. In this article, we propose an approach allowing object identification from statecharts that are themselves established from use cases. Firstly, and after a hierarchical decomposition of the system into its controlled parts and controlling subsystem, we convert the natural language description of the use cases into statecharts, where states and events on transitions refer to the systemcontrolled units.
During statechart establishment, ambiguities, inconsistency and impreciseness are removed. Secondly, without any premature commitment to design, we identify objects that directly concern each controlled unit, and we identify objects of other types according to their categorization. In doing so, we follow the separation of concerns principle $[14,15]$. Once objects have been identified, classes to which those objects belong can be determined. In this way, our approach is consistent with the Rumbaugh's bottom-up method to discover inheritance links and organize classes [16].

It is worth noting here that statecharts are precise models, easy to understand and work with. They can be used in all the phases of the development process, and hence they constitute a highly reused artifact.

Based on statecharts, our approach not only allows objects identification from use cases in a systematic manner, but also provides the benefit of an improved requirements specification. The identified objects, in particular those that are related to the domain entities allow for enriching use cases and making them clearer, more adequate and useful. Furthermore, the presented method is easy to apply, integrates nicely with existing software development processes, such as the unified process [4], and does not impose an inappropriate overhead.

The rest of the article is organized as follows: section 2 describes the UML models used in our approach, namely, the use cases models, the UML statechart with its extension for representing the embedded systems behavior, and the object model with the object categorization. Section 3 presents our object identification process. In section 4 , we provide an overview of the related works and compare them with our approach. Finally, in section 5 the conclusion and future directions of our research are outlined.

\section{Embedded Systems Modeling with UML}

An embedded system consists of a controlling subsystem and controlled units [17]. A controlling subsystem is a set of computer systems, while the controlled units can be any of the broad range of systems with mechanical behavior, any device, from a simple blender to a complex robot. Typically, a controlling subsystem performs control operations to receive input 
from the environment and/or sends commands to the controlled units appropriately.

An embedded system can, therefore, be decomposed into its controlling subsystem and controlled units. To control these units, the controlling subsystem may perform computation on their status information, from which it determines the necessary commands to send to these units and then updates this status information [18]. To facilitate the necessary object identification, we perform a hierarchical decomposition strategy. According to Glinz [13], a good decomposition is one that follows the basic software engineering principle of information hiding and separation of concerns. This decomposition will provide more clarity and preciseness to the use case descriptions and the converted statecharts, where actions and events will be related to these controlling units, such as door closed, elevator stopped in an elevator control system.

In the following, we present the use case elements, the UML statechart as well as its extension to effectively model the features of embedded systems, and finally the object categories relevant to these systems.

\subsection{Use Cases}

To specify the ways in which a user uses a system, a use case captures who (actor) does what (interaction) with the system and for what purpose (goal) [2]. An actor is often a human user (see example in Figure 1). In embedded systems, an actor can be an external $\mathrm{I} / \mathrm{O}$ device or a timer. External I/O devices and timer actors are particularly prevalent in embedded systems [6]. A complete set of use cases specifies all different ways to use the system, and therefore defines all behavior required of the system. Since use cases serve as a means of communication between developers and users, they are

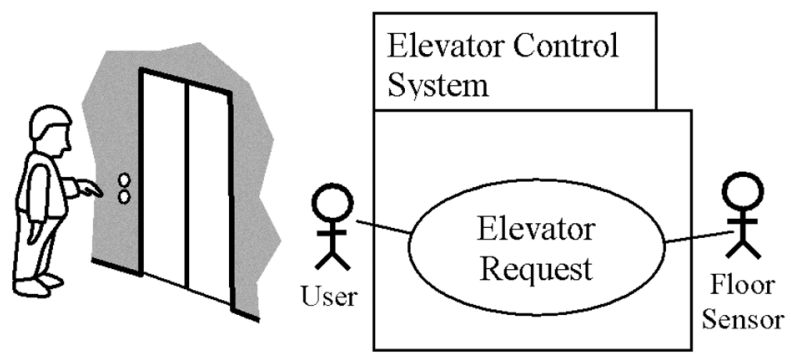

Fig. 1. A use case diagram for an elevator request. fundamentally written in simple text. However, their textual description presents some drawbacks such as the lack of precision and conciseness.

On the other hand, every large system needs to be decomposed in order to make it comprehensible and manageable. Therefore, like in RT-UML [5], the system use cases may be broken down into sub(system-level)-use cases using "include" and "extends". The system use case is then realized by the set of subsystems collaborating together. Each subsystem is specified in terms of its subsystem level use case.

In our approach, we are interested in the description of a use case defined by a name, actor, preconditions, postconditions, normal steps, and alternative steps according to Cockburn's template [19]. To this template we have added a quality of service section in which we describe non-functional requirements (response time, security, cost, accuracy, etc.). Figure 2 illustrates a typical textual description of a request elevator use case in an elevator control system.

Thus, a use case can be seen as a tuple $<$ ucName, ucActor, ucPre, ucPost, ucSteps, ucAlt, ucQoS $>$ with ucName a label that uniquely identifies a use case, ucActor a primary actor and the secondary actors, ucPre a set of preconditions, ucPost a set of postconditions, ucSteps a set of ordered normal steps, ucAlt a set of alternative steps, and ucQoS a set of qualities of services.

Each step in ucSteps is a tuple $<$ sNumber, sOper $>$ with sNumber a step number, sOper an operation (actor action(s) or system response $(\mathrm{s})$ ). An operation may also be a branching statement to another step. A normal step may be associated with a set of alternative steps.

An alternative step can be seen as a tuple $<$ altStepNumber, guardCond, altStepOper $>$, with altStepNumber an alternative step number and guardCond a guard condition on this step, and altStepOper an alternative operation. The latter may be divided into several sub-operations. A subset of use case steps in an automated teller machine system may be as follows:

$\{<1$, User inserts card $>;<2$, System Asks for PIN $>,<2 \mathrm{a}$, [invalid card] $>,<2 \mathrm{a} 1$, System emits alarm $>,<2 \mathrm{a} 2$, System ejects card $>\}$. 
Use Case Name: Request elevator.

Context of Use: The elevator system has many elevators that service many users at any one time, taking them from one floor to another.

Primary Actor: User, Secondary actor: Floor sensor

Precondition: User is at a floor and wants an elevator.

Postcondition: Elevator has arrived at the floor in response to user request.

\section{Description:}

1. User presses an up floor button. The system selects an elevator to visit this floor.

2. If the elevator is idle, the system determines in which direction the elevator should move in order to service the new request.

3. The system commands the elevator door to close. After the door has been closed, the system commands the elevator to start moving, either up or down.

4. As the elevator moves between floors, the floor sensor detects that the elevator is approaching a floor and notifies the system.

5. The system checks whether the elevator should stop at this floor. If so, the system commands the elevator to stop.

6. When the elevator has stopped, the system commands the elevator door to open.

7. If there are no other outstanding requests, the elevator stays at the current floor with the door open.

Alternatives:

1a. User presses down floor button to move down. The system response is the same for the main sequence.

2a. The elevator is moving, Go to step 4.

5a. Current floor is not in the list of the floor to visit, Go to step 4.

7a. When there are other outstanding requests, Go to step 2 .

Quality of Service:

1. Elevator movement must be minimized.

2. Use case response time must be minimized.

3. Doors must be closed before starting any elevator movement

Fig. 2. Textual description of the elevator request Use Case.

\subsection{Statecharts}

Statecharts, conceived as a visual formalism for the design of reactive systems [20], extend finite state diagrams by hierarchy, concurrency, and communication, which, besides the timing constraints, are fundamental features of embedded systems.

An UML statechart diagram consists of a finite number of states and transitions between states (Figure 3a). Actions are executed either on the transition between states or on the entry into the state. A state is a stage in the behavior pattern of an entity. Modeling substates makes sense when an existing state also exhibits complex behavior, thereby motivating to explore its substates. States are shown as rounded rectangles.

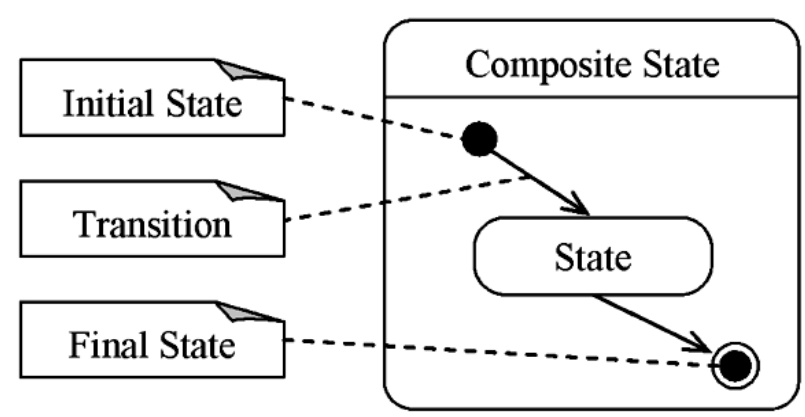

Fig. 3a. Example of UML statechart syntax.

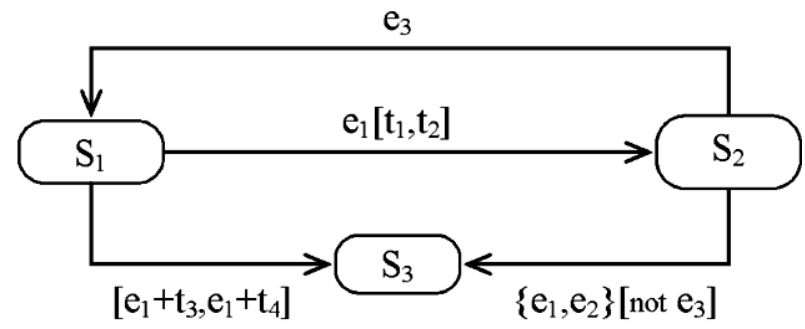

Fig. 3b. Example of extended UML statechart.

A transition is a progression from one state to another and will be triggered by an event, that is either internal or external, to the entity being modeled. A guard is a condition that must be true in order to traverse a transition. The transition is only taken if the named event occurs and the guard evaluates to true. All these transition elements are optional. The transition is shown as an arrowed line (see Figure 3a). 
Although it is a valuable means for modeling embedded systems behavior, UML statechart still needs some extensions to make it both enough expressive to represent the features of these systems and enough precise to support activities like object identification, property verification, test generation, etc. [21]. A detailed description and justification of these extensions is beyond the scope of this work. Figure $3 b$ illustrates these extensions that we summarize as follows:

1. Guards can make reference to events, indicating whether an event has occurred.

2. Associate a set of events to a transition, indicating that the transition is triggered by the concurrent occurrence of the set of events. For example, the transition from $S_{2}$ to $S_{3}$ is enabled only when the events $\mathrm{e}_{1}$ and $\mathrm{e}_{2}$ occur, and in the absence of the event $\mathrm{e}_{3}$.

3. Associate a time interval to a transition, indicating that the transition occurs at a time (not known a-priori) belonging to the temporal interval.

4. A time interval can specify a time window as an expression of event occurrence. For example, the transition from $S_{1}$ to $S_{3}$ can be triggered not earlier than $t_{3}$ time units after the occurrence time of the event $\mathrm{e}_{1}$, and not later than $t_{4}$ time units after the occurrence time of that event.

\subsection{Object Modeling and Categorizing}

At the requirements analysis level, objects are usually determined from use cases according to certain categorization. Jacobson et al. have divided the analysis space in three orthogonal dimensions: information, behavior and presentation [22]. This object categorization is useful to control the system's specification complexity, creating a multidimensional modeling space to allow a multiple-view analysis of the requirements with adequate semantic references. The same categorization framework was adopted by Fernandes and Machado in [8], with some emphasis on control objects as a crucial component in any embedded system.

For the purpose of identifying objects from use cases in embedded systems, we adapt the object categories described in [6] for including coordination, application logic and timer objects, too. We present this categorization as follows:
1. Interface object: handles the exchange between the system and its environment. An interface object must be encapsulated in such a way that if a change is made to the exchange between the system and its environment, only the interface object has to be modified, leaving other objects unchanged.

2. Entity object: Long-living object that stores information (typically the entities in entityrelationship models). The entire behavior associated to the manipulation of that information must be included in the entity object. Typically, an entity object is accessed by many use cases.

3. Coordination object. It is an overall decisionmaking object that determines overall sequencing for a collection of related objects inherent to a use case. It does not encapsulate any computation other than the one needed for the coordination. Its main responsibility is to supervise other objects in order to achieve the use case goal.

4. Application logic object: It contains the details of the application logic. It is needed to hide the application logic from the data being manipulated (because it is likely that the application logic could change regardless of the data).

5. A timer object: encapsulates temporal conditions and triggers activities in other objects, periodically or at appropriate time points.

6. A control object: encapsulates appropriate computations that involve a group of entity objects or that cannot be naturally associated to other objects.

We believe that this categorization of objects makes models more stable, in the sense that modifications made during the system development are easier to locate and affect a smaller number of objects. Moreover, this categorization leads to the application of the separation of concerns principle early in the system development lifecycle. Separation of concerns principle makes the specification easier to understand, more stable and reusable $[14,15]$. 


\section{Object Identification Process}

Based on the observation that an embedded system is a controlling subsystem and a set of controlled units, we first perform a hierarchical decomposition of the system into its parts. This allows for better comprehension of the use case and facilitates determining evolution state of the controlled parts regarding the underlying use case. Afterward, we derive the statechart in an iterative and incremental way (main feature of the unified process).

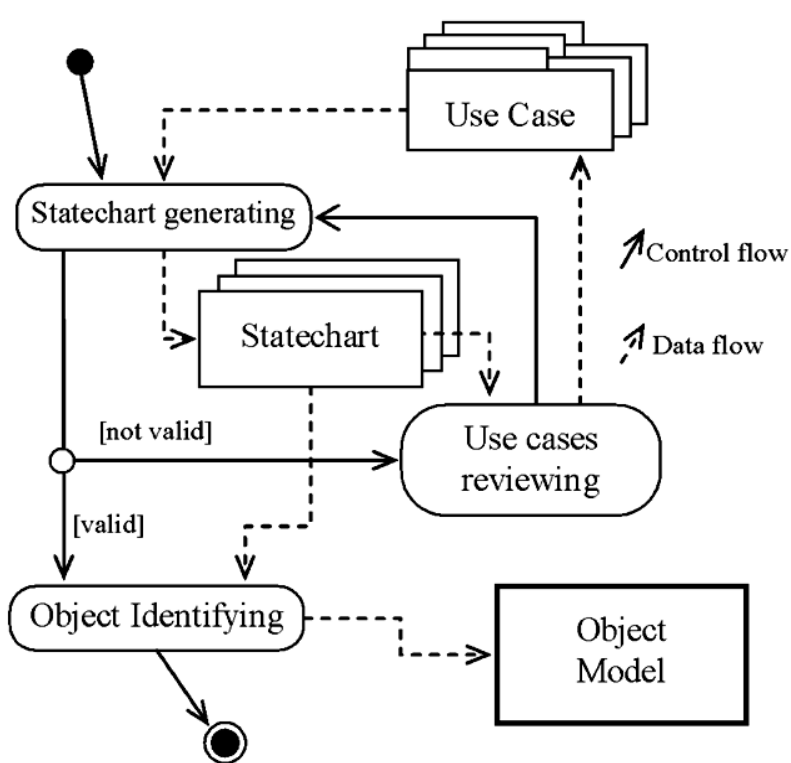

Fig. 4. Object identification process.

Figure 4 illustrates our approach to identification of objects from use cases after decomposing the system into its parts. We first need to circumvent the drawbacks of the textual description use cases and give rise to the elements that do constitute the objects, such as operations and data items. To this end, instead of directly using the natural language in use case description, we transform it into a statechart, according to the appropriate procedure described in section 3.1. During this step, the use case text may be reviewed and modified in order to remove ambiguities and to exhibit more preciseness and completeness.

Second, the obtained statechart is used with the object categorization in embedded systems, to determine the objects according to the appropriate procedure described in section 3.2.

\subsection{From Use Cases to Statecharts}

In contrast to other approaches to derive statecharts from use cases, such as $[3,9,12,23,24]$, we do not restrict the textual description of use case for capturing the requirements. Informal notations are good for recording requirements at an early stage, when great expressiveness and ease of use is more important than formal correctness and executability. Table 1 describes our procedure to transform a use case text into a statechart. This procedure is structured into four iterations as described below:

1. For each use case, build a graph of behavior sequences (GBS) where each node corresponds to a step and each edge links two nodes that correspond to the two consecutive steps in the use case.

2. Model the normal flow first, integrate the alternative flows later and place the steps' guards on the corresponding edges.

1. Transform the GBS into a statechart. Each node is transformed into a state and the link is transformed into a transition.

2. Specify the operations of each step in the entry of the corresponding state.

3. Specify explicitly the necessary operations related to the guards in the corresponding states.

4. For each operation, specify the necessary state variables.

5. Specify event names and possible guards on the corresponding transitions.

1. Group the states, corresponding to steps that may be performed in parallel, into a superstate.

2. If possible, decompose each state that incorporates more than one operation, into substates such that:

- Operations blocks that may be performed in parallel should be specified in different parallel substates,

- Only one actor or one system unit should be referred in a substate,

- Guarded operations block should be specified in a separate substate.

3. Represent abstract use cases as hierarchical statecharts. 


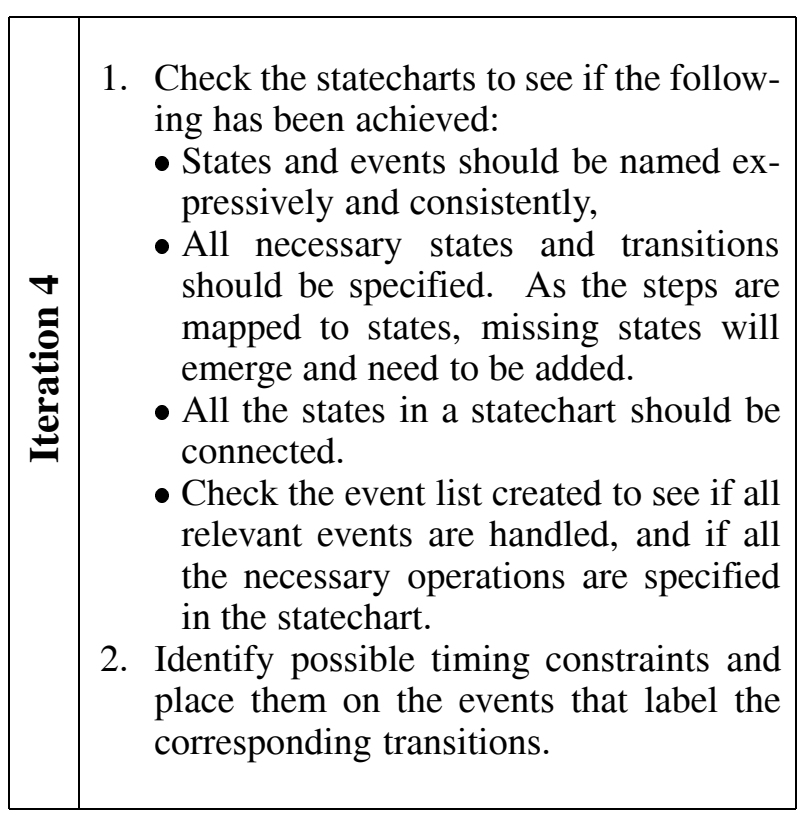

Table 1. Statechart derivation procedure.

Iteration 1: Transforming the use case into a Graph of Behavior Sequences (GBS).

Iteration 2: Transforming the GBS into a statechart.

Iteration 3: Refining and restructuring the statechart.

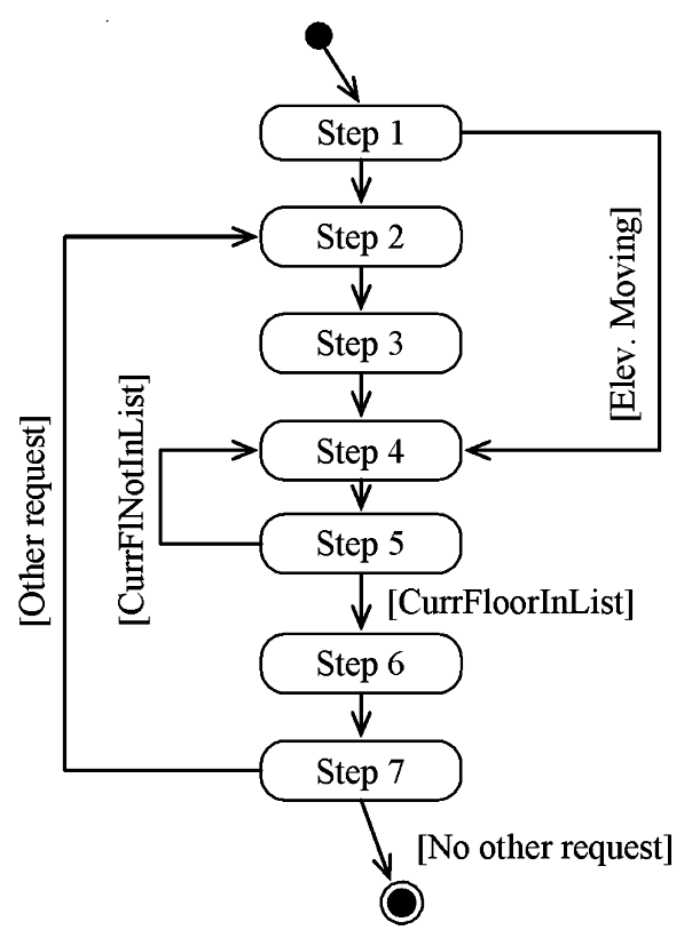

Fig. 5. Graph of the behavior sequence of an elevator request use case.
Iteration 4: Checking the statechart for internal completeness and consistency, and identifying the possible timing constraints.

In Iteration 1, a use case is transformed into a GBS (Figure 5), which is a graph where each node corresponds to a use case's step and the edges link the use case's ordered steps. In Iteration 2, we transform the GBS into a statechart (Figure 6). The nodes become states and the

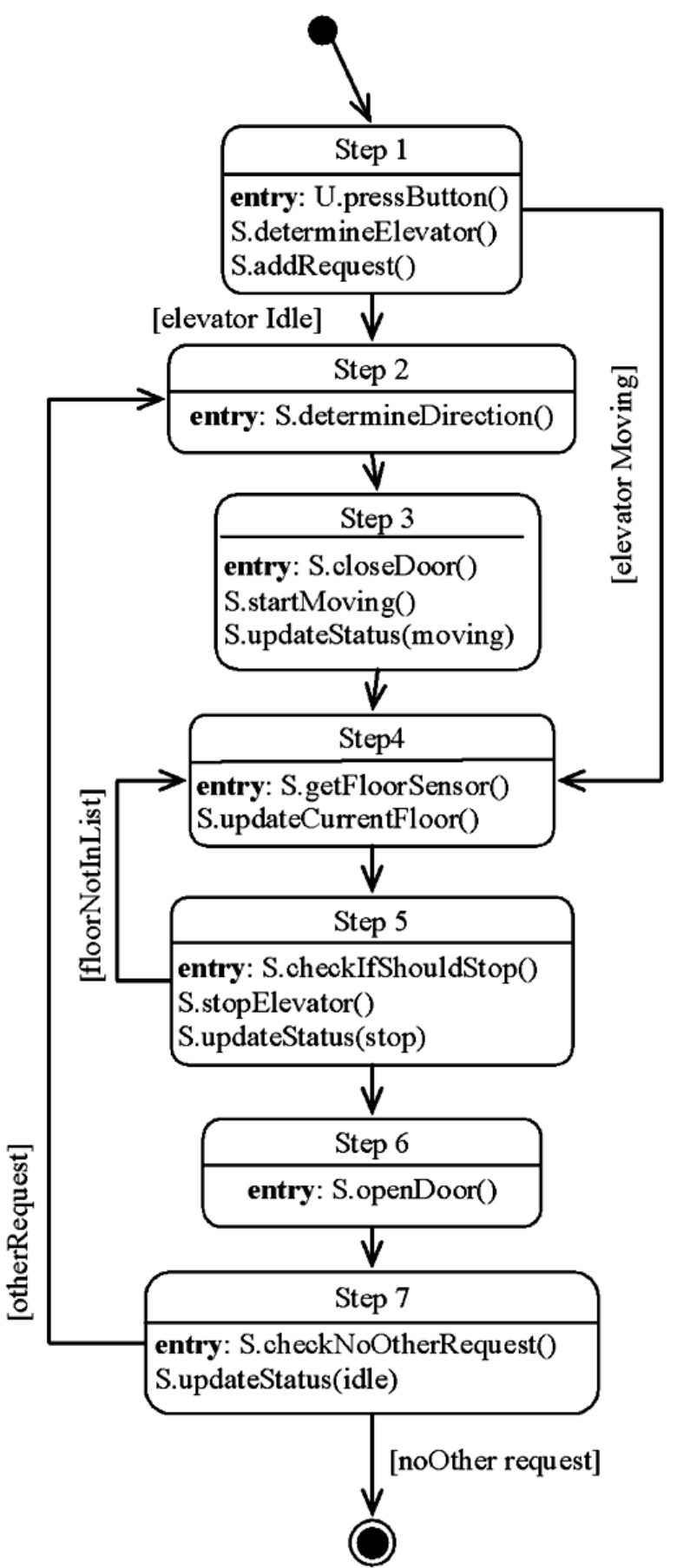

Fig. 6. Statechart after iteration 2. 
edges become transitions. In the entry of each state, we specify corresponding actor's actions or system's responses, according to the general format: "sAct_id.Oper", with sAct_id an actor identifier to distinguish between actor's action $(\mathrm{U})$ and system's response (S), and Oper the operation name. In Iteration 3 , we restructure the statechart in order to raise possible concurrency and hierarchy. Finally, in Iteration 4, we check the statechart for consistency and completeness.

In the following section, we discuss how operations are determined from each use case's step and show how to attain final version of the statechart.

Step 1: Two operations are specified: 1) user presses an up floor button, and 2) system selects an elevator. This last operation specifies that the system should select an elevator to visit the source floor. This description is ambiguous, in the sense that it isn't precisely defined. Should the system determine any elevator, or should it determine the most suitable one?

To determine the most suitable elevator in a multiple-elevators control system while taking into account the specified quality of service "minimize the elevator movement", the system must have, not only the source floor number (sfl) and the desired direction (dd) to be supplied by the user, but also some state information about each elevator (idle, moving up, moving down, last visited floor).

Therefore, the first two data items should be associated with the event "button pressed". During the statechart elaboration we uncover the ambiguity related to the described operation of "selecting an elevator" and provide the necessary precision by determining events and data items.

Step 2: In the entry of the corresponding state, we specify the operation "S.determineDirection".

Step 3: Two operations are explicitly specified in this step: "closeDoor" and "startMoving". They correspond to the system commands of closing the door and starting to move elevator. However, commanding the elevator to start moving should be followed by updating its status. Therefore, the missing operation "updateStatus()" should be added to the corresponding state of this step. In doing so, we uncover an omission in the use case. In addition, for the sake of security, the elevator must start moving not earlier than at certain time units after the door has been closed. This important timing constraint is not explicitly specified in this use case. In doing so, we uncover a possible temporal inconsistency.

Step 4: the secondary actor "floor sensor" detects the fact that the elevator is approaching a floor that becomes current, and notifies the

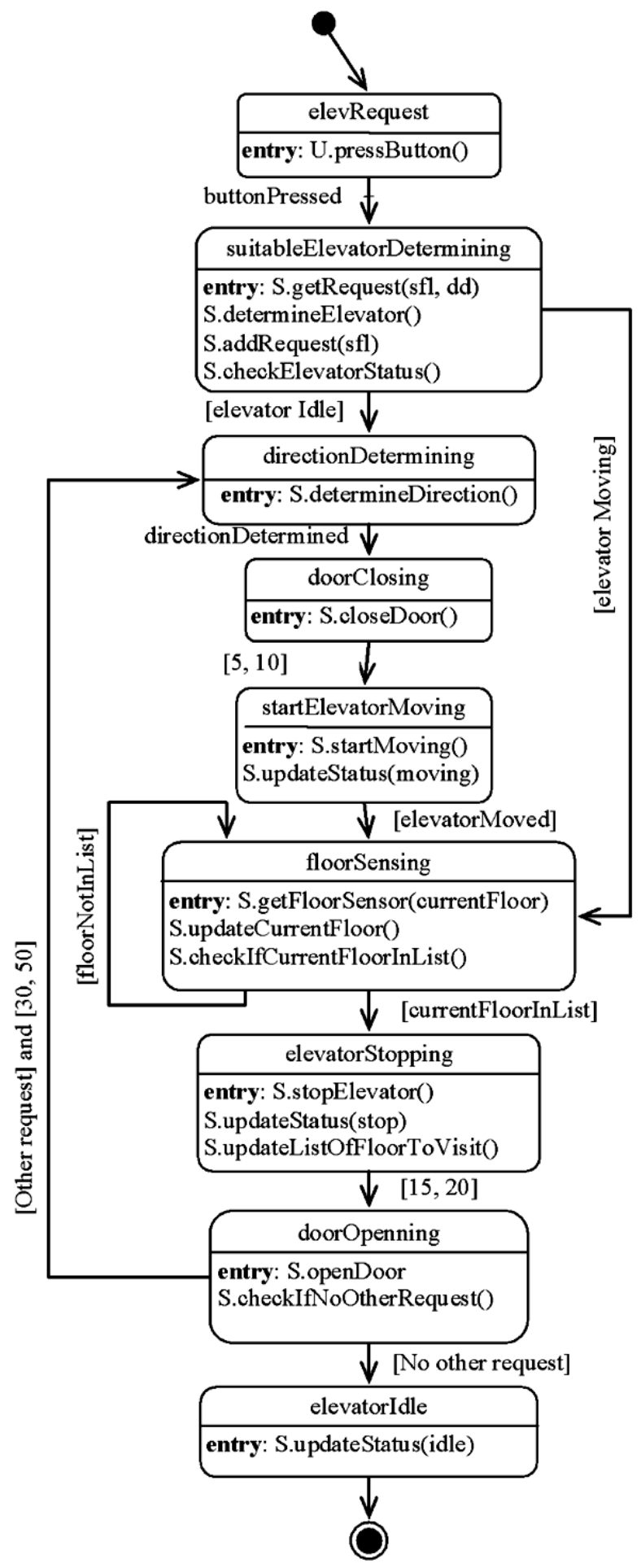

Fig. 7. Statechart after iteration 4. 
system. The system updates the current floor information of the appropriate elevator.

Step 5: The system checks if the current floor is in the list of the floors to visit by the elevator concerned. However, this operation needs access to all the pending requests, including the one that is submitted by the user at the first step. When examining the previous steps, we do not find any reference to adding this request. To uncover this omission in the use case, we add the operation "addRequest" in the first state, after the operation "determineElevator". This state will then comprise three operations rather than two.

Step 6: The system commands the door to open. We therefore place the operation "openDoor" in the corresponding state entry.

Step 7: The system checks if there are other requests. If so, the door must wait for a certain time before closing. This temporal requirement is not explicitly defined in the use case.

Therefore, we explicitly specify this timing constraint as a label on the transition that starts from the door opening state and ends at the direction determining state. If there are no other requests, the elevator stays at the current floor with the door open. However, the information status of this elevator that becomes idle must be updated. We therefore specify the action "updateStatus(idle)". Figure 7 illustrates the final version of the statechart obtained after applying iterations 3 and 4 .

\subsection{From Statechart to Objects}

The derived statechart modeling states of the system's controlled units, incorporates the necessary elements to define objects, namely, actions and data items. First, for each controlled unit, we determine the necessary objects according to the procedure defined in Table 2.

Let's apply this procedure to our resulting statechart (Figure 7) in order to determine objects and distribute responsibilities amongst them. The controlled units identified from the statechart are a set of elevators, a set of elevator doors, and floor sensors. For the sake of brevity, we do not consider other units such as buttons and lamps. In the following, we discuss the objects identification from the statechart:

1) We define for the whole use case, a coordination object (named elevRequestCoordinator).
1. For the whole use case, define a coordinator object.

2. Define an interface object for each actor or controlled unit.

3. Define an entity object for each controlled unit that is referred to by at least one action or guard. Every action that refers to only this controlled unit should be assigned to its defined entity object.

4. When an action involves a group of controlled units, define a control object. Every action that involves this controlled unit set should be assigned to its defined control object.

5. Define a timer object for the action set that is triggered in specific time intervals or at a specified time point.

6. Define an application logic object to incorporate actions that cannot be handled by any other object category.

7. Check the statechart to verify if all specified actions have been distributed to objects.

Table 2. Object identification procedure.

This object does not encapsulate any functional action. It only triggers actions encapsulated in other objects or receives signals from them.

2) For each controlled unit and actor that receives and/or sends events from/to the system, we define an interface object to receive inputs from these units or actor, and/or to send outputs or commands to them. In our example, we define interface objects for the identified controlled units (elevators, doors, and the floor sensors).

For instance, for every elevator, we define, an interface object "elevatorInterface" to receive commands (startMoving(), stopElevator()) from the system. Also, for every elevator door, we define an interface object "doorInterface" to receive commands (openDoor, closeDoor), and for the floor sensor, we define the interface object "floorSensorInterface", to handle interaction at every elevator approaching a floor.

3) The actions such as addRequest(), and updateStatus() refer to an elevator. We therefore define an entity object, named "elevStatusPlan", for each elevator. 
Moreover, the guard condition "elevator idle" invokes an action that checks if the elevator is idle. From this guard condition, we identify the attribute "elevator status", which may have the value "idle". This attribute should also be encapsulated by the entity object "elevStatusPlan".

This entity object should provide information on whether the elevator is moving or idle, as well as on the current floor if it is at a floor or the last floor, if it is moving between floors. Furthermore, the action "addRequest ()" should also be assigned to this entity object. The latter should therefore encapsulate the list of floors to visit.

As there are no computation actions, nor guards referring to the controlled units "door" and "floor Sensor", we do not specify any entity object to these controlled units.

4) The action "determineElevator()" that selects the most suitable elevator to service the user request, needs access to all elevator entity objects to use the current status of the elevators. It, therefore, corresponds to a control object named "Scheduler".

5) Timing constraints related to door events will be handled by the timer object "doorTimer". This object sends a timeout signals to the use case coordinator object according to the specified timing constraint.

6) Finally, qualities of service mentioned in the use case text are mainly related to the optimization of the elevator movement. Usually, to deal with some of these non-functional requirements, we need an object that periodically calculates statistical parameters such as: average busy time and idle time of each elevator, floor(s) where elevator(s) are very often requested. To this end, we define an application logic object "statisticalMeasure" that encapsulates such computations.

Figure 8 depicts the object model obtained from the statechart by applying the procedure described in Table 2. As previously noted, the coordinator object "elevRequestCoordinator" is an overall decision-making object that determines overall sequencing for all the objects related to the use case. All these objects are therefore linked to it. Moreover, the control object "Scheduler" has links to the objects "elevStatusPlan", since it accesses each elevator entity object in order to use its state information, to

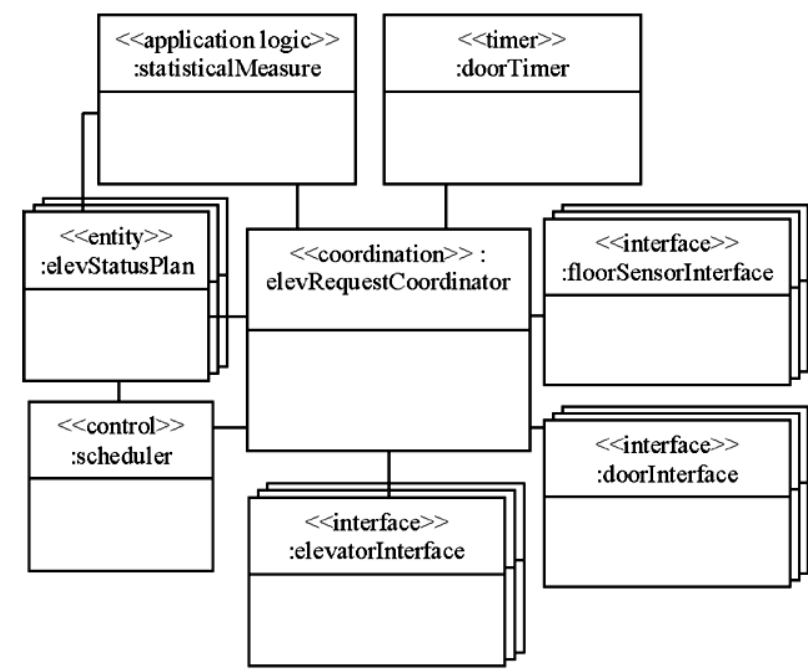

Fig. 8. The object model for the elevator request use case.

select the most suitable elevator to the user request. The object "statisticalMeasure" has links to the "elevRequestCoordinator" and "elevStatusPlan" objects.

\section{Related Works}

In recent years, object-oriented techniques have been employed in the development of embedded systems, e.g. RT-UML [5], UML-RT [3], OCTOPUS [25], Artisan Real-Time Perspective [26], etc. There are also several papers in the literature that discuss the process of building object model, e.g. $[8,9,11,12,22,27]$.

In UML-RT (UML for Real-Time), which has been included in UML 2.0 [28], the construction of object and class diagrams is performed by the derivation of scenario diagrams and capsule collaboration from use case scenarios, and by the synthesis of object and class diagrams from initial sequence diagrams that only depict interaction between control software and system devices. Software objects are determined using CRC technique [2] or Jacobson's object categories (interface, control, and entity) [22].

In RT-UML and Artisan Real-Time perspective, objects are identified by the textual analysis of problem statements or use case descriptions. In Octopus, The system under study is partitioned into subsystems. An analysis phase is performed for each subsystem whose object/class model is built from the problem description, by underlying nouns to determine candidate 
classes. Usually, too many objects/classes are obtained in that manner. This technique is also applied in $[3,9,12]$, but with the use of automatic tools for natural language analysis of the use case text and the problem statements.

In [27], Rosenberg and Scott extend the approach of Jacobson et al. [22] for identifying objects, using robustness analysis. However, their steps describe, in a very brief form, what needs to be done to put together the main parts of the object diagram. These describing steps do not give any type of detailed instruction on "how".

In [8], an approach to identify objects from use cases is presented. It consists of a 4-step rule set. The approach defines the steps to obtain a holistic set of objects. Each use case is transformed in three objects (one interface, one data, and one control). Besides the restricted types of objects, this approach presents some limitations in a sense that it concentrates on what needs to be done rather than addressing "how" it can be done.

In [11], an approach that identifies classes is presented. It is based on goals of use cases, without descriptions. The approach produces use case entity diagrams as a vehicle for deriving classes from use cases. However, only domain classes are identified and there is no more global technique that would allow making the transition between the two models in a systematic manner.

Most of the above mentioned tools and methods begin with building classes rather than objects. Our approach focuses on objects and communication between them rather than on the classes and associations between them. Since most real-time and embedded applications have a static structure, it is convenient to see the system under development as a set of communicating objects rather than as a set of classes with associations. To some extent, our approach complements the existing ones in the sense that, at the analysis stage, it begins with the dynamic model instead of the structural one. We believe that it is better to build the dynamic model before the static one. If one begins with the dynamic model, it is easier to identify operations, events and also attributes, which will be needed in the static model, and to obtain no more than what is needed.

\section{Conclusion}

In this paper we have presented a systematic approach to transition from the use cases to the object model in the embedded systems area. Our approach presents a technique for converting use cases into statecharts and uses the latter as a means to identify objects. The semi-formal nature of statecharts allows for discovering the necessary objects and their properties (operations and attributes), which are needed for realizing the use case.

The derived statecharts help the developers in uncovering ambiguities, omissions, impreciseness, and inconsistency that may be present in the natural language description of the use case. In this way, while preserving the advantages of the use cases' natural language description (expressiveness and ease to use), we also allow for using existing tools to verify and prove some properties of embedded systems.

We are currently in the process of developing a semi-automatic and interactive system that helps synthesizing statechart diagrams from use cases and building the object model. In addition, we are investigating the subject of modifying the XMI DTD to represent our extended statechart by means of XML documents, in order to automatically generate the interaction models.

\section{Acknowledgment}

We would like to thank Professor Zoubir Mammeri from Paul Sabatier University in Toulouse (France) whose suggestions on the first draft of this document were very helpful. Furthermore, we would like to thank the anonymous referees whose comments were useful in improving this paper.

\section{References}

[1] A.G. Sutcliffe, N.A.M. Maiden, S. Minocha, D. MANUAEL, Supporting scenario-based requirements engineering, IEEE Transaction on Software Engineering, 12 (1998), pp. 1072-1088.

[2] I. SOMMERVILLE, Software engineering, 6th edition, Addison-Wesley, 2001.

[3] B. SELIC, Using UML for modeling complex realtime systems, LNCS, 1474 (1998), pp. 250-262. 
[4] I. JaCOBSON, G. BoOCH, J. RuMBAUGH, The unified software development process, Addison-Wesley, 1999.

[5] B.P. DougLASS, Real-time UML: Developing efficient objects for embedded systems, 2nd edition, Addison-Wesley, 2000.

[6] H. GomAA, Designing concurrent, distributed, and real-time applications with UML, Addison-Wesley, 2000.

[7] G. Booch, J. Rumbaugh, I. Jacobson, The unified modeling language user's guide, Addison Wesley, 1999.

[8] J.M. FERnANDES, R.J. MACHADO, From use cases to objects: An industrial information systems case study analysis, Proceedings of the 7th Int'l Conference on Object-oriented Information Systems, (2001), Calgary, Canada, pp. 319-328.

[9] R.S. WAHANO, B.H. FAR, A framework for object identification and refinement process in objectoriented analysis and design, Proceedings of the 1st Int'l Conference on Cognitive Informatics, (2002), Calgary, Canada.

[10] M.T. Kimour, Generative sequence diagrams for requirements specification in real-time systems, Proceedings of the 2nd ACS/IEEE Int'l Conference on Computer Systems and Applications, (2003), Tunis, Tunisia.

[11] Y. LIANG, From use cases to classes: a way of building object model with UML, International Journal of Information Software and Technology, 2 (2003), pp. 163-180.

[12] D. LiU, K. Subramaniam, B.H. FAR, A. EberLein, Automatic transition from use cases to class model, Proceedings of the IEEE Canadian Conference on Electrical and Computer Engineering, (2003), Montréal, Canada, pp. 831-834.

[13] M. Glinz, S. Berner, S. Joos, J. Ryser, The ADORA approach to object-oriented modeling of software, Proceedings of the 13th Int'l Conference on Advanced Information Systems Engineering LNCS, 2068 (2001), Inderlaken, Switzerland, pp. 76-92.

[14] G. Kiczales, J. Lamping, A. MendheKar, C. MAEDA, C.V. LOPES, J.-M. LOINGTIER, J. IRWIN, Aspect-oriented programming, Proceedings of the 11th Int'l European Conference on Object-oriented Programming, (1997), Finland, LNCS 1241, pp. 140-149.

[15] H. OSSHER, P. TARR, Using multidimensional separation of concerns to (re)shape evolving software, Communications of the ACM, 10 (2001), pp. 43-50.

[16] J. Rumbaugh, M. Blaha, W. Premerlani, F. EDDY, W. LARENSON, Object-oriented modeling and design, Prentice Hall, 1991.

[17] T.M. ChUnG, H.G. DIETZ, Language constructs and transformation for hard real-time systems, $A C M$ SIGPLAN Notices, 11 (1995), pp. 41-49.
[18] M.T. KIMOUR, Real-time object-oriented program restructuring for improved schedulability, Proceedings of the 8th Int'l Conference on Real-Time Systems, (2000), Paris, France.

[19] A. COCKBURN, Writing effective use cases, Addison Wesley, 2001.

[20] D. HAREL, Statecharts: a visual formalism for complex systems, Science of Computer Programming, 8 (1987), pp. 231-274.

[21] V.D. Bianco, L. LavazZa, M. Maury, A formalization of UML statecharts for real-time software modeling, Proceedings of the Int'l Workshop on Integrated Design and Process Technology, (2002), Pasadena, CA, USA.

[22] I. JACOBSOn, M. Christerson, P. Jonsson, G. ÖVERGAARD, Object-oriented software engineering: A use case driven approach, Addison-Wesley, 1992.

[23] H. BEHRENS, Requirements analysis and prototyping using scenarios and statecharts, Proceedings of the Int'l Workshop on Scenarios and State Machines: Models, Algorithms and Tools, (2002), Orlando, Florida, USA.

[24] S.S. SomÉ, Beyond scenarios: Generating state models from use cases, Proceedings of the Int'l Workshop on Scenarios and State Machines: Models, Algorithms and Tools, (2002), Orlando, Florida, USA.

[25] M. AWAD, J. KuUSELA, J. ZIEGLER, Object-oriented technology for real-time systems: A practical approach using OMT and Fusion, Prentice Hall, 1996.

[26] ARTiSAN Software Tools - Modeling Solutions for Real-Time Software and Embedded Systems Development. http://www . artisansw. com, last visited July 2004.

[27] D. Rosenberg, K. ScOTT, Use case driven object modeling with UML: A practical approach, Addison-Wesley, 1999.

[28] Object Management Group. Unified modeling language: superstructure. Version 2.0. OMG Adopted Specification ptc/03-08-02. www . uml . org, 2004.

Received: January, 2004 Revised: July, 2004 Accepted: July, 2004

Contact address: Mohamed T. Kimour Laboratoire de Recherche en Informatique Université de Annaba

23000, Annaba Algérie

e-mail: kimour@yahoo.com

Djamel Meslat Laboratoire de Recherche en Informatique Université de Annaba BP 12

23000, Annaba Algérie

e-mail: meslati_djamel@yahoo.com 
MOHAMED T. KimOUR is an assistant professor at the Department of Computer Science at the University of Annaba. His research interests include requirements engineering and model-based development of embedded real-time systems.

DJAMEL MESLATI is the head of the research group on evolution and reuse of software systems. His research interests include software development and evolution methodologies, and separation of concerns models. 